\title{
Intravascular large B-cell lymphoma associated with myelofibrosis: A case report
}

\author{
JONG GWON CHOI ${ }^{1}$, HWAN HWI CHO ${ }^{1}$, SANG ROK KANG ${ }^{1}$, SE MIN JANG ${ }^{2}$, \\ EUN HYUNG YOO ${ }^{3}$, HYUN JUNG CHO ${ }^{3}$, SUN MOON KIM ${ }^{4}$ and DO YEUN CHO ${ }^{1}$
}

\author{
Departments of ${ }^{1}$ Oncology-Hematology, ${ }^{2}$ Pathology, ${ }^{3}$ Laboratory Medicine and ${ }^{4}$ Gastroenterology, \\ Konyang University Hospital, Daejeon 35365, Republic of Korea
}

Received November 9, 2016; Accepted March 14, 2017

DOI: $10.3892 /$ mco.2017.1398

\begin{abstract}
Myelofibrosis (MF) is often accompanied by chronic myeloid leukemia, hairy cell leukemia, or certain primary myeloproliferative neoplasms, but is rarely associated with lymphoid neoplasms. We herein describe a case of intravascular large B-cell lymphoma (IVLBCL) with MF. IVLBCL is a rare, aggressive type of extranodal B-cell lymphoma, defined by proliferation of lymphomatous cells within small-to medium-sized vessels. A 60-year-old woman was admitted to the hospital with anemia, thrombocytopenia and fever. Bone marrow biopsy findings included trilineage hematopoiesis, increased numbers of immature cells, markedly abnormal and enlarged megakaryocytes, and diffuse fibrosis in multiple focal areas throughout the entire bone marrow space. When the patient was first hospitalized, hepatosplenomegaly was not present. Although initially considered during differential diagnosis, an aggressive lymphoma could not be diagnosed prior to colonoscopy, which was conducted 4 weeks after admission. A biopsy of the terminal ileum revealed IVLBCL with cells with atypical nuclei. Immunophenotyping of the atypical large cells yielded a positive result for CD79a and negative results for terminal deoxynucleotidyl transferase, myeloperoxidase, CD3, CD10, CD20, B-cell lymphoma (Bcl)-2, Bcl-6 and cytomegalovirus. The patient was diagnosed with IVLBCL complicated by MF. This case may serve as a reminder that IVLBCL may be the cause of secondary MF.
\end{abstract}

\section{Introduction}

Intravascular large B-cell lymphoma (IVLBCL) is a rare type of extranodal diffuse large B-cell lymphoma (DLBCL) (1), which is characterized by the proliferation of lymphoma cells

Correspondence to: Professor Jong Gwon Choi, Department of Oncology-Hematology, Konyang University Hospital, 158 Gwanjeodong-ro, Seo-gu, Daejeon 35365, Republic of Korea E-mail: jabuss@naver.com

Key words: intravascular B-cell lymphoma, myelofibrosis, thrombocytopenia in the lumina of small vessels and capillaries, clinically aggressive behavior, usually with an unfavorable prognosis. In the majority of the cases, IVLBCL has extended to involve several regions at diagnosis. This disease was previously referred to as malignant angioendotheliomatosis; however, when the lymphoid origin of the malignant cells was identified, it was renamed intravascular malignant lymphomatosis $(2,3)$. The precise mechanism underlying the distinctive behavior of this lymphoma remains unknown.

Although IVLBCL is typically associated with a rapid, aggressive course and short survival, a few exceptions of long-term survivors have been reported (4). The clinical manifestations of IVLBCL are diverse, as the majority of signs and symptoms are related to the organ most extensively affected. Furthermore, clinical differences may vary according to the geographical origin of the patients (5). Patients with IVLBCL in Western countries are usually predisposed to a high frequency of central nervous system and skin involvement, whereas Asian patients exhibit a tendency towards bone marrow involvement, thrombocytopenia, fever and haemophagocytic syndrome; however, these different clinical patterns do not affect patient survival. Variation in other clinical parameters does not affect prognosis, except for presence of the cutaneous variant, which is associated with a better prognosis. However, the cutaneous variant of IVLBCL is very rare in Asia (3\%) compared with Western countries (24\%), and patients with this variant present with particular clinical characteristics (6).

Laboratory findings are not usually specific, but may raise suspicion regarding a diagnosis of IVLBCL. Approximately $65 \%$ of patients with IVLBCL have anemia. Furthermore, an increase in serum lactate dehydrogenase (LDH) and $\beta 2$-microglobulin levels is observed in $>80 \%$ of the patients. Monoclonal gammopathy is reported in $14 \%$ of the patients and hepatic, renal, or thyroid function is affected in $15-20 \%$ of the patients. Therefore, such tests are generally used in the staging workup for IVLBCL. The conventional staging system frequently leads to a high proportion of false negatives when using various imaging methods, such as whole-body computed tomography. This may be due to the fact that IVLBCL mainly occurs without identifiable tumor masses or lymphadenopathy $(5,6)$.

Myelofibrosis (MF) is a chronic myeloproliferative neoplasm characterized by the accumulation of reticulin and 
collagen fibers, as well as the proliferation of fibroblastic mesenchymal cells in the bone marrow. These changes are associated with increases in neoangiogenesis and circulating levels of particular cytokines, including transforming growth factor (TGF)- $\beta 1$, platelet-derived growth factor (PDGF) and fibroblast growth factor (FGF)- $\beta$ (7). MF may be classified as primary idiopathic or secondary type at the onset or during the course of the disease. Secondary MF is commonly observed in acute megaloblastic leukemia, chronic myeloid leukemia, hairy cell leukemia and myelodysplastic syndrome. However, secondary MF is rare in malignant lymphoma.

We herein report a case of IVLBCL associated with MF, which improved following chemotherapy for the lymphoma.

\section{Case report}

A 60-year-old woman presented at the Konyang University Hospital (Daejeon, South Korea) on March 10, 2016, with fever that started 1 month prior and thrombocytopenia identified at a local clinic. At the time of admission, the patient had a blood pressure of $120 / 70 \mathrm{mmHg}$, a heart rate of 88 beats per min, a respiratory rate of 20 breaths per min and a temperature of $39^{\circ} \mathrm{C}$. There was no significant previous medical history of hypertension, diabetes mellitus, or tuberculosis. On physical examination, multiple ecchymoses were observed over the lower extremities bilaterally, with dried blood in the pharynx and gums and edema of the lower lip. However, there was no enlargement of cervical or inguinal lymph nodes. The patient's abdomen was mildly distended but soft, non-tender, without enlarged organs or palpable masses. The results of other examinations were unremarkable.

The laboratory tests revealed a white blood cell (WBC) count of $6,900 / \mu 1$, a hemoglobin level of $7.3 \mathrm{~g} / \mathrm{dl}$ and a platelet count of $26,000 / \mu 1$. The WBCs comprised $55 \%$ segmented neutrophils, $27 \%$ lymphocytes, $12 \%$ monocytes, $1 \%$ eosinophils and $1 \%$ basophils. The reticulocyte count was within the normal range. Leukemic blasts were not observed. Coagulation tests revealed a prothrombin time of $14.8 \mathrm{sec}$ and an activated partial thromboplastin time of $32.3 \mathrm{sec}$. The fibrinogen level was $350 \mathrm{mg} / \mathrm{dl}$, the D-dimer value was $2.1 \mu \mathrm{g} / \mathrm{ml}$ and the antithrombin activity $72 \%$ (not suggestive of disseminated intravascular coagulation). Peripheral blood examination revealed normocytic and normochromic red blood cells, no polychromasia, a normal WBC count with no toxic granulation or vacuolations, and a decreased platelet count. The serum LDH level was 832 U/I (normal range, 120-240 U/I). The total bilirubin level was $1.04 \mathrm{mg} / \mathrm{dl}$. The results of other blood tests, including creatinine and bicarbonate, and liver function tests were unremarkable. In immunoassay tests, the patient tested negative for antibodies against Hantaan virus, rickettsia, leptospira, scrub typhus and severe fever with thrombocytopenia syndrome virus. The differential diagnosis also included fulminant infection or autoimmune disease, which were excluded by laboratory and microbial examinations.

On computed tomography (CT) examination, the liver, pancreas, spleen and kidneys were normal. The presence of thoracic or abdominal involvement was excluded. Bone marrow examination using pelvic trephine biopsy and aspiration resulted in 'dry tap'. Bone marrow aspiration slides revealed no particles or peripheral dilution, and immature

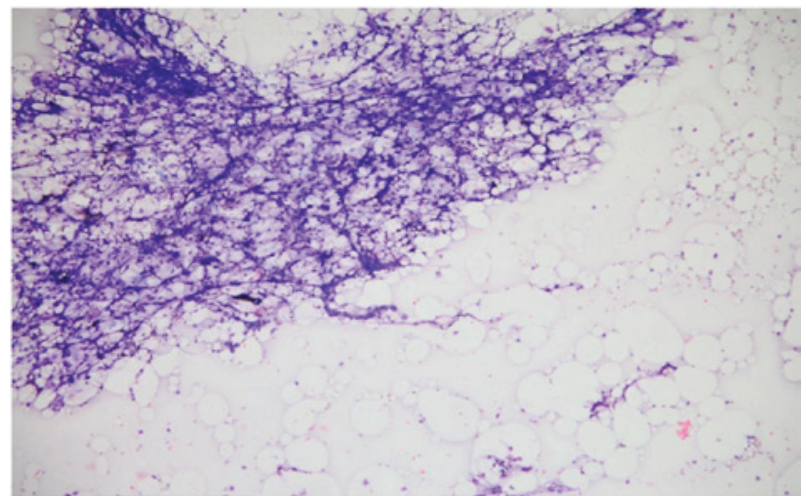

Figure 1. Bone marrow aspirate showing a lack of particles and peripheral dilution. Immature cells were occasionally identified (Wright-Giemsa staining; magnification, x200).

A

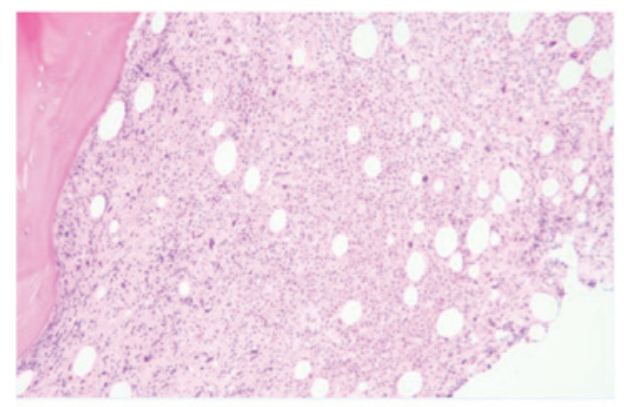

B

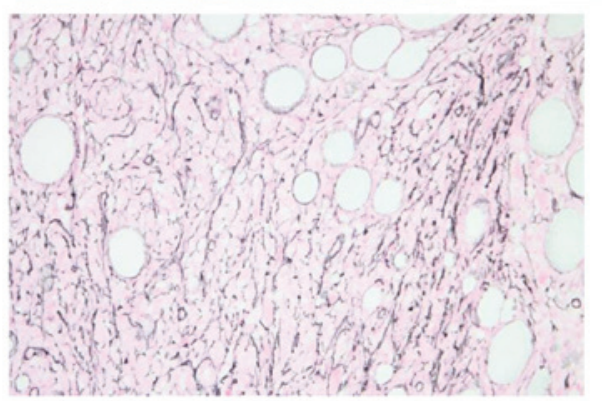

Figure 2. (A) Trephine bone marrow biopsy showing $~ 60 \%$ cellularity, with increased atypical megakaryocytes and fibrosis (hematoxylin and eosin staining; magnification, x200). (B) Reticulin staining of bone marrow biopsy revealed grade 3 reticulin fibrosis (reticulin staining; original magnification, x400).

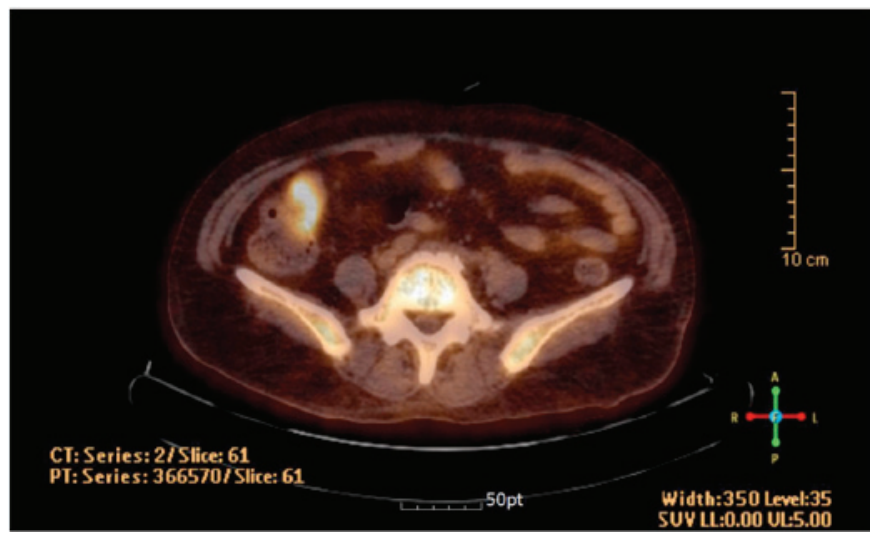

Figure 3. Integrated ${ }^{18} \mathrm{~F}$-fluorodeoxyglucose positron emission tomography-computed tomography revealed focal hypermetabolism in the terminal ileum [maximum standardized uptake value $\left(\mathrm{SUV}_{\max }\right)=5.9$; delayed $\left.\mathrm{SUV}_{\max }=7.8\right]$ 

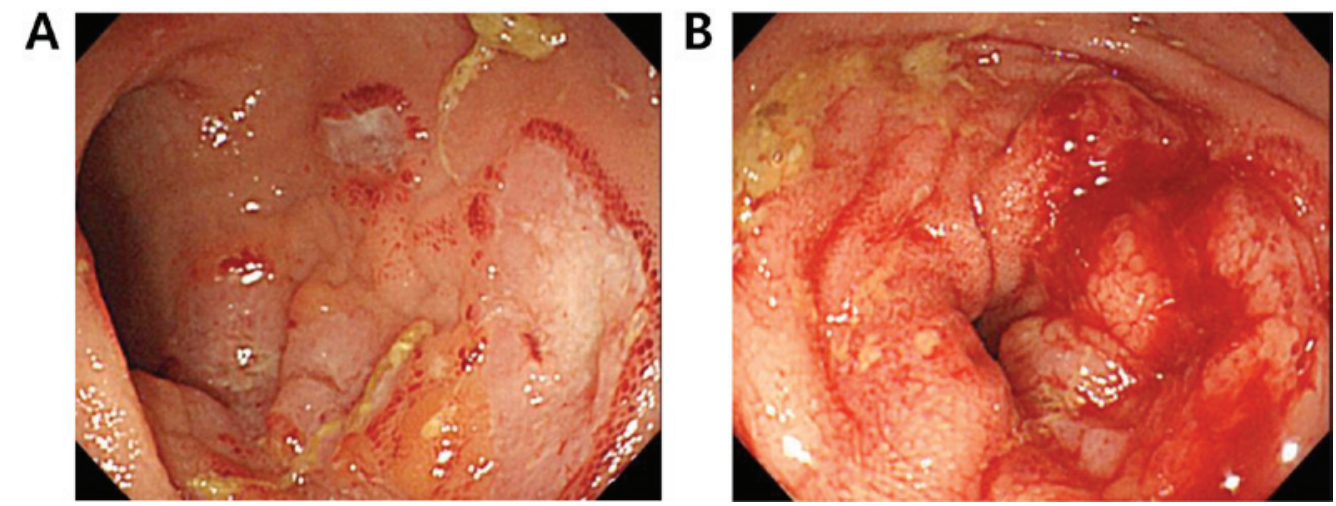

Figure 4. Colonoscopy revealed (A) severe colitis with cherry-red spoterythema and (B) easy contact bleeding in the cecum.

A

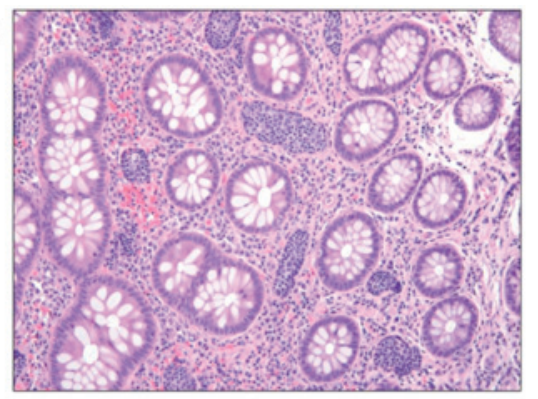

C

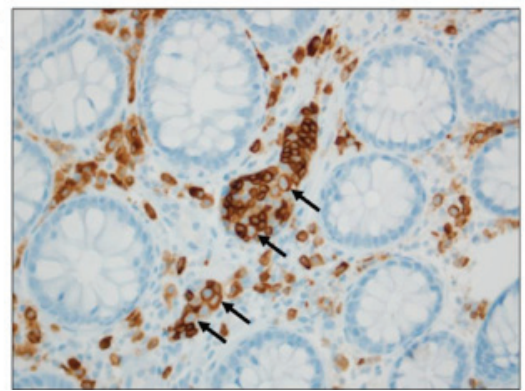

B

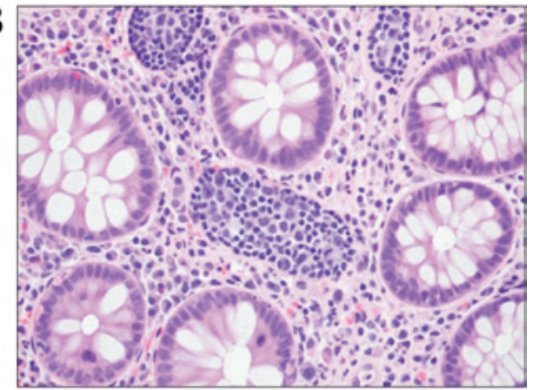

D

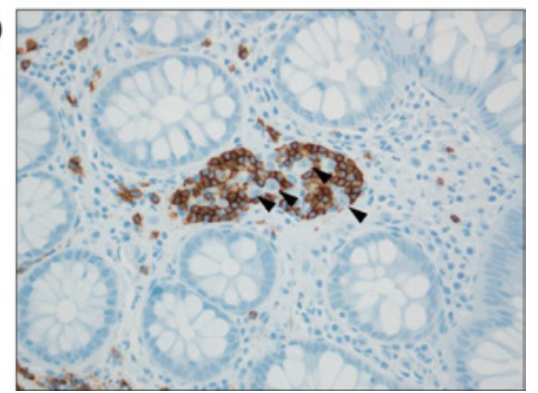

Figure 5. Photomicrographs of intravascular large B-cell lymphoma. (A) The small blood vessels of the terminal ileal mucosa were filled with lymphoid cells [hematoxylin and eosin (H\&E) staining; magnification, x200). (B) Atypical large lymphoid cells with prominent nucleoli were observed intermingled with small lymphocytes in the vascular lumina with occasional mitotic figures (H\&E staining; magnification, $\mathrm{x} 400)$. The atypical large cells were (C) immunoreactive for CD79a (arrows), but (D) negative for CD20 (arrowheads); magnification, x400.

cells were occasionally identified (Fig. 1). Bone marrow biopsy showed an estimated cellularity of $\sim 60 \%$, which was considered mildly hypercellular for the patient's age. In cellular areas, trilineage hematopoiesis was observed, along with increased numbers of immature cells. There were increased numbers of megakaryocytes, which were markedly abnormal, with various shapes and increased size. There was deviation from the normal nuclear: Cytoplasmic ratio and an abnormal pattern of chromatin. Diffuse fibrosis was observed in multiple focal areas throughout the bone marrow space (Fig. 2). The pathological diagnosis from bone marrow biopsy recommended excluding post-essential thrombocythemia MF. Furthermore, JAK2 V617F mutation and immunoglobulin gene rearrangement were not detected.

Although the patient had been treated with antibiotics, she had recurrent fever. Blood and urine cultures were negative. An integrated ${ }^{18} \mathrm{~F}$-fluorodeoxyglucose positron emission tomography/CT scan of the skull to mid-thigh revealed an enlarged and hypermetabolic terminal ileum, with a maximum standardized uptake value of 7.2 (Fig. 3). Subsequently, the patient underwent total colonoscopy; this examination ruled out ulcerative colitis or tuberculous colitis of the terminal ileum (Fig. 4). On microscopic examination of the terminal ileum, the mucosal architecture was relatively well-preserved. However, there were numerous lymphoid cells present in the lumina of small blood vessels. The lymphoid cells in the small vessels of the mucosa included large cells with atypical nuclei and prominent nucleoli, and occasional mitotic figures. The cells were solely lodged in the lumina of the vessels and absent from the lamina propria. Immunohistochemical analysis revealed that the large cells were positive for CD79a, but negative for CD3, CD20, terminal deoxynucleotidyl transferase and myeloperoxidase. The histopathological findings suggested the diagnosis of IVLBCL (Fig. 5).

Two days after receiving the pathology report from the colonoscopic biopsy, the patient abruptly developed hyperbilirubinemia (peak total bilirubin, $16.5 \mathrm{mg} / \mathrm{dl}$ ). A CT scan revealed hepatomegaly and an enlarged spleen, and advancing 
transmural thickening of the wall of the terminal ileum due to the lymphoma.

Based on the clinical findings, the patient was diagnosed with an IVLBCL with MF. The lymphoma was classified as clinical stage IV. Subsequently, the patient underwent chemotherapy with cyclophosphamide $1,250 \mathrm{mg}$, day 1 ; doxorubicin $83 \mathrm{mg}$, day 1; vincristine $2 \mathrm{mg}$, day 1; and prednisone $100 \mathrm{mg}$, days 1-5 (CHOP regimen). The B symptoms resolved, with additional resolution of hyperbilirubinemia and a reduction in the size of the liver. However, the patient developed drowsiness 7 days after the first cycle of chemotherapy, as well as pneumonia with pancytopenia. Granulocyte colony-stimulating factor and intravenous antibiotic injections were administered; however, the patient succumbed to the disease 15 days after the first cycle of chemotherapy.

\section{Discussion}

Chronic idiopathic MF, also referred to as as primary idiopathic MF, may be described as a disorder of myelopoietic tissue relating to abnormal cloning of hematopoietic stem cells in the bone marrow accompanied by fibrosis (replacement of cells in the marrow with scar tissue), which impairs the production of normal blood cells (8). MF is often accompanied by an enlarged spleen and/or liver, inadequate numbers of platelets, gout and high uric acid levels. Secondary MF is observed in association with various systemic diseases, including metastatic carcinoma, tuberculosis, osteoporosis and toxic marrow injury following chemical exposure or irradiation $(9,10)$. Secondary MF may also occur in various hematological diseases, such as myelodysplastic syndrome, hairy cell leukemia, chronic myeloid leukemia and acute megakaryoblastic leukemia (11). However, secondary MF is rare in malignant lymphoma and cases of MF associated with IVLBCL are extremely scarce.

IVLBCL is an aggressive, rare extranodal lymphoma that is characterized by the localization of lymphomatous cells in small- to medium-sized vessels; it is also recognized as a subset of DLBCL in the 2008 WHO classification (12).

IVLBCL may affect any organ and it is likely to have already spread widely at the time of diagnosis. Various symptoms and signs may appear, depending on its distribution. These may be either non-specific and generalized (e.g., B symptoms, elevated LDH level and cytopenia) or confined to the affected organ (e.g., cutaneous lesions or hepatic insufficiency). This variation leads to a significant range of differential diagnoses, including neoplastic, infectious and autoimmune disorders. The lack of lymph node involvement and mass lesions further confounds the clinical picture and delays diagnosis. Generally, the lymphoma cells are large with round, readily distinguishable nuclei. Furthermore, mitotic activity is high. However, rare cases with smaller cells or anaplastic morphology have been reported.

IVLBCL may also involve the sinusoidal spaces of the bone marrow, liver and spleen. This predilection for vascular spaces has been hypothesized to be secondary to a defect in homing receptors on cells, such as a lack of CD29 ( $\beta 1$-integrin) and CD54 (intercellular adhesion molecule-1) adhesion molecules (13). In particular, involvement of the bone marrow may be difficult to detect, as the neoplastic cells are diffuse rather than aggregated, and their morphology may mimic that of hematopoietic precursors, as seen in the case presented herein. Malignant cells uniformly express pan-B-cell antigens (CD20, CD79a) and may also express other antigens, such as CD5 (38\%) and CD10 (13\%).

Little is known on the correlation between MF and lymphoma, except for information provided by the few reported cases of MF with concomitant or subsequent lymphoma (14). Although lymphoma is a well-known cause of $\mathrm{MF}$, it is uncommon. Gisselbrecht et al reported no patients with MF in a series of 1,883 patients with diffuse aggressive non-Hodgkin lymphoma (15). In addition, the pathogenesis of the fibrotic changes in the bone marrow of patients with IVLBCL was unknown until recently. In the majority of MF cases, megakaryocytes with multilobulated nuclei proliferate and exhibit clustering in the marrow (16). These megakaryocytes have abnormally located P-selectin in their intracytoplasmic vacuoles and demarcation membrane system, leading to increased emperipolesis of neutrophils (17). Neutrophils release enzymes that cause megakaryocytes to secrete various cytokines, including TGF- $\beta$, PDGF and FGF, from their $\alpha$-granules (18). These cytokines play an essential role in the development of stromal proliferation. PDGF induces fibroblast proliferation, while TGF- $\beta$ promotes synthesis and accumulation of extracellular matrix proteins, including fibronectin, type I and III collagens, and endothelial cells, causing neoangiogenesis $(19,20)$. Production of osteoprotegerin by stromal and endothelial cells leads to unbalanced osteoblast proliferation, resulting in osteosclerosis and neoangiogenesis (21). Therefore, MF is considered to be a clonal disorder of a hematopoietic stem cell and fibrosis is considered to be a secondary change caused by the release of cytokines from progenitor cells (22).

In cases of lymphoma with secondary MF, PDGF is mostly synthesized in megakaryocytes, but may also be secreted by cells of the monocyte-macrophage lineage (23). Furthermore, $\mathrm{T}$ cells secrete TGF, which may cause fibrosis. The plasma TGF- $\beta$ levels were found to be elevated in cases of MF associated with peripheral T-cell, cytotoxic T-cell and splenic marginal zone lymphomas (24-27). TGF- $\beta$ was elevated prior to treatment in a patient with DLBCL and significantly decreased following treatment, and was associated with advanced stage, a high-risk international prognostic index and bulky disease (28). Furthermore, Munoz et al reported overexpression of Smad1 in lymphoma cells, and phosphorylation of Smad1 is mediated by TGF- $\beta$ in the microenvironment of lymphoma cells; they found that activation of Smad1 by TGF- $\beta$ induced non-Hodgkin lymphoma cell proliferation (29). The findings presented herein suggest that DLBCL cells produce cytokines, such as PDGF and TGF- $\beta$, which cause their morphological change into spindle-shaped cells.

The case presented herein highlights several pitfalls in the recognition and diagnosis of IVLBCL. The major symptoms were non-specific: Fever of unknown origin, multilineage cytopenia and hepatosplenomegaly. Although bone marrow biopsy was initially performed, the burden of disease at that time was low and the morphology was ambiguous. Therefore, certain characteristics led to misdiagnosis as primary MF. When the disease eventually caused severe hepatomegaly and hyperbilirubinemia, the lymphoma cells were present in 
greater numbers and were clearly recognizable as lymphomatous, particularly in the liver, but less so in the marrow. Of note, although hepatomegaly and hyperbilirubinemia were considered to be due to the IVLBCL infiltration of the liver, this was not proven pathologically. However, these manifestations improved following the initiation of chemotherapy, despite the delay in diagnosis.

In the present case, the patient developed MF and IVLBCL simultaneously, and these were treated with chemotherapy. The presentation of MF was similar to that of IVLBCL, suggesting that IVLBCL plays an important role in the pathogenesis of MF. Certain cytokines are hypothesized to stimulate the growth of fibroblasts and the synthesis of collagen by bone marrow fibroblasts. Further studies and additional case reports are required to elucidate the pathogenesis of secondary MF and improve our understanding of the immunological dysregulation associated with IVLBCL. A search for lymphoid malignancy should be considered when the cause of MF is unclear.

\section{References}

1. Gatter KC and Warnke RA: Intravascular large B-cell lymphoma In: Jaffe ES, Harris NL, Stein H and Vardiman JW (eds): World Health Organization Classification of tumours: Pathology and Genetics of Tumours of Haematopoietic and Lymphoid Tissues. International Agency for Research on Cancer (IARC), Lyon, IARC Press, pages, pp177-178, 1991.

2. Sepp N, Schuler G, Romani N, Geissler D, Gattringer C, Burg G, Bartram CR and Fritsch P: 'Intravascular lymphomatosis' (angioendotheliomatosis): Evidence for a T-cell origin in two cases. Hum Pathol 21: 1051-1058, 1990.

3. Sheibani K, Battifora H, Winberg CD, Burke JS, Ben-Ezra J, Ellinger GM, Quigley NJ, Fernandez BB, Morrow D and Rappaport H: Further evidence that 'malignant angioendotheliomatosis' is an angiotropic large-cell lymphoma. $\mathrm{N}$ Eng $\mathrm{J}$ Med 314: 943-948, 1986.

4. Ferreri AJ, Campo E, Seymour JF, Willemze R, Ilariucci F, Ambrosetti A, Zucca E, Rossi G, López-Guillermo A Pavlovsky MA, et al: Intravascular lymphoma: Clinical presentation, natural history, management and prognostic factors in a series of 38 cases, with special emphasis on the "cutaneous variant'. Br J Haematol 127: 173-183, 2004.

5. Murase T, Yamaguchi M, Suzuki R, Okamoto M, Sato Y, Tamaru J, Kojima M, Miura I, Mori N, Yoshino T and Nakamura S: Intravascular large B-cell lymphoma (IVLBCL): A clinicopathological study of 96 cases with special reference to the immunophenotypic heterogeneity of CD5. Blood 109: 478-485, 2007

6. Murase T, Nakamura S, Kawauchi K, Matsuzaki H, Sakai C, Inaba T, Nasu K, Tashiro K, Suchi T and Saito H: An asian variant of intravascular large B-cell lymphoma: Clinical, pathological and cytogenetic approaches to diffuse large B-cell lymphoma associated with haemophagocytic syndrome. Br J Haematol 111: 826-834, 2000

7. Rameshwar P, Oh HS, Yook C, Gascon P and Chang VT: Substance p-fibronectin-cytokine interactions in myeloproliferative disorders with bone marrow fibrosis. Acta Haematol 109: $1-10,2003$

8. Tefferi A: Primary myelofibrosis: 2014 update on diagnosis, risk-stratification, and management. Am J Hematol 89: 915-925, 2014.

9. Viallard JF, Parrens M, Boiron JM, Texier J, Mercie P and Pellegrin JL: Reversible myelofibrosis induced by tuberculosis. Clin Infect Dis 34: 1641-1643, 2002

10. Atasever T, Vural G, Yenidünya S, Ataoğlu O, Atavci S and Unlü M: Tc-99mMIBI bone marrow uptake in bone marrow fibrosis secondary to metastatic breast carcinoma. Clin Nucl Med 22: 655-656, 1997.

11. Fu R, Yu H, Wu YH, Liu H and Shao ZH: Hodgkin's lymphoma associated with myelofibrosis: A case report. Oncol Lett 10 $1551-1554,2015$.
12. Nakamura S, Ponzoni M and Campo E: Intravascular large B-cell lymphoma. In: Swerdlow SH, Campo E, Harris NL, et al (eds): WHO Classification of Tumours of Haematopoietic and Lymphoid Tissue. Lyon, France, IARC Press, pages, pp252-253, 2008.

13. Ponzoni M, Arrigoni G, Gould VE, Del Curto B, Maggioni M, Scapinello A, Paolino S, Cassisa A and Patriarca C: Lack of CD29 (betal integrin) and CD54 (ICAM-1) adhesion molecules in intravascular lymphomatosis. Hum Pathol 31: 220-226, 2000.

14. Gabali AM, Jazaerly T, Chang CC, Cleveland R and Kass L: Simultaneous hepatosplenic T-cell lymphoma and myelofibrosis. Avicenna J Med 4: 34-36, 2014.

15. Gisselbrecht C, Gaulard P, Lepage E, Coiffier B, Brière J, Haioun C, Cazals-Hatem D, Bosly A, Xerri L, Tilly H, et al: Prognostic significance of T-cell phenotype in aggressive non-Hodgkin's lymphomas. Grouped'Etudes des Lymphomes de l'Adulte (GELA). Blood 92: 76-82, 1998.

16. Briere J, Kiladjian JJ and Peynaud-Debayle E: Megakaryocytes and platelets in myeloproliferative disorders. Baillieres Clin Haematol 10: 65-88, 1997.

17. Schmitt A, Jouault H, Guichard J, Wendling F, Drouin A and Cramer EM: Pathologic interaction between megakaryocytes and polymorphonuclear leukocytes in myelofibrosis. Blood 96: 1342-1347, 2000

18. Tefferi A: Myelofibrosis with myeloid metaplasia. N Engl J Med 342: 1255-1265, 2000

19. Cervantes F and Martinez-Trillos A: Myelofibrosis: An update on current pharmacotherapy and future directions. Expert Opin Pharmacother 14: 873-884, 2013.

20. Charni Chaabane S, Coomans de Brachène A, Essaghir A, Velghe A, Lo Re S, Stockis J, Lucas S, Khachigian LM, Huaux F and Demoulin JB: PDGF-D expression is down-regulated by TGF $\beta$ in fibroblasts. PLoS One 9: e108656, 2014

21. Chagraoui H, Tulliez M, Smayra T, Komura E, Giraudier S, Yun T, Lassau N, Vainchenker $W$ and Wendling $F$ : Stimulation of osteoprotegerin production is responsible for osteosclerosis in mice overexpressing TPO. Blood 101: 2983-2989, 2003.

22. Jacobson RJ, Salo A and Fialkow PJ: Agnogenic myeloid metaplasia: A clonal proliferation of hematopoietic stem cells with secondary myelofibrosis. Blood 51: 189-194, 1978.

23. Wang JC, Chang TH, Goldberg A, Novetsky AD, Lichter S and Lipton J: Quantitative analysis of growth factor production in the mechanism of fibrosis in agnogenic myeloid metaplasia. Exp Hematol 34: 1617-1623, 2006

24. Okabe S, Miyazawa K, Lguchi T, Sumi M, Takaku T, Ito Y, Kimura Y, Serizawa H, Mukai K and Ohyashiki K: Peripheral T-cell lymphoma together with myelofibrosis with elevated plasma transforming growth factor-beta1. Leuk Lymphoma 46: 599-602, 2005

25. Kikukawa M, Umahara T, Kikawada M, Kanaya K, Sakurai H, Shin K, Mori M and Iwamoto T: Peripheral T-cell lymphoma presenting as myelofibrosis with the expression of basic fibroblast growth factor. Geriatr Gerontol Int 9: 395-398, 2009.

26. Abe Y, Ohshima K, Shiratsuchi M, Honda K, Nishimura J, Nawata $\mathrm{H}$ and Muta K: Cytotoxic T-cell lymphoma presenting as secondary myelofibrosis with high levels of PDGF and TGF-beta. Eur J Haematol 66: 210-212, 2001.

27. Matsunaga T, Takemoto N, Miyajima N, Okuda T, Nagashima H, Sato T, Terui T, Sasaki H, Ohmi N, Hirayama Y, et al: Splenic marginal zone lymphoma presenting as myelofibrosis associated with bone marrow involvement of lymphoma cells which secrete a large amount of TGF-beta. Ann Hematol 83: 322-325, 2004.

28. Bedewy AM,Elgammal MM,Bedewy MM andEl-Maghraby SM: Assessing DcR3 expression in relation to surviving and other prognostic factors in B cell non-Hodgkin's lymphoma. Ann Hematol 92: 1359-1367, 2013.

29. Munoz O, Fend F, de Beaumont R, Husson H, Astier A and Freedman AS: TGFbeta-mediated activation of Smad1 in B-cell non-Hodgkin's lymphoma and effect on cell proliferation. Leukemia 18: 2015-2025, 2004. 